\title{
A theory of heterogeneous city growth*
}

\author{
Christian GHIGLINO \\ Department of Economics, University of Essex, UK \\ Kazuo NISHIMURA \\ RIEB, Kobe University, Japan \\ and \\ Alain VENDITTI \\ Aix-Marseille Univ., CNRS, EHESS, Centrale Marseille, AMSE \\ \& EDHEC Business School, France
}

\begin{abstract}
We consider an economy with three cities producing different outputs. Two cities produce intermediate goods, a type 1 producing an intermediate "agricultural" good with capital and labor only, and a type 2 producing an intermediate "industrial" good with capital, labor and human capital, and the last type 3 city produces the final good which is obtained from the two intermediate goods and labor. The asymmetric introduction of human capital allows us to prove that the three cities experience at the equilibrium heterogeneous endogenous growth rates which are proportional to the growth rate of human capital. We show that the "industrial" type 2 city is characterized by the larger growth rate while the "agricultural" type 1 city experiences the lower growth rate, and thus the type 3 city is characterized by a growth rate which is a convex combination of the two formers. This implies that the relative size in terms of output of the "agricultural" city decreases over time. This property allows to recover the empirical fact that most nonagricultural production occurs in growing metropolitan areas. But, simultaneously, as we prove that total labor employed in each city is proportional to the total population, the relative population size distribution of cities is constant over time as shown in empirical studies.
\end{abstract}

Keywords: Urban dynamics, human capital, endogenous growth, heterogeneous growth rates, city inequalities

Journal of Economic Literature Classification Numbers: C61, C62, O41, R11, R12.

${ }^{*}$ This work was supported by French National Research Agency Grants ANR-08BLAN-0245-01 and ANR-17-EURE-0020, and by the Japan Society for Promotion of Science, Grants-in-Aid for Research \#15H05729 and \#16H0233598. 


\section{Introduction}

Since the seminal contribution of Black and Henderson [4], the endogenous growth theory has been used to understand urban dynamics. Two facts appear to be central in such an analysis. First, the relative population size distribution of cities is extremely stable over time although most countries have experienced periods of industrialization and urbanization. Indeed, for any country, the rank of a city according to population multiplied by its population is constant. Such a stylized fact, well established by Eeckout [8] who shows that the size distribution of cities is log-normal, appears to hold across many countries and time periods (see Soo [13], Eaton and Eckstein [7]). Second, most nonagricultural production in developed countries occurs in metropolitan areas (see Black and Henderson [3]). Our objective in this paper is to show with a simple model that these two properties can hold simultaneously. While external effects and increasing returns through knowledge spillovers have been shown to be important to discuss economic activity agglomeration, we consider a very simple framework with no externality and constant returns. However, we focus on the crucial role of human capital which is as in Lucas [11] the engine for endogenous growth.

We consider an economy with three cities which are characterized by different outputs. Two cities over three produce intermediate goods. A type 1 city produces an intermediate "agricultural" good with capital and labor only, and a type 2 city produces an intermediate "industrial" good with capital, labor and human capital. We assume indeed that industrial production requires more qualified workers. The type 3 city produces the final good which is obtained from the two intermediate goods and labor. This asymmetric introduction of human capital allows to prove that the three cities will at the equilibrium experience heterogeneous growth rates. Proceeding that way, we adapt the formulation of Acemoglu and Guerrieri [1] and, following the strategy of Ghiglino et al. [9], we obtain endogenous growth through a standard mechanism based on human capital.

We prove that endogenous growth occurs in all cities, and that all the growth rates are proportional to the growth rate of human capital. The 
"industrial" type 2 city is characterized by the larger growth rate while the "agricultural" type 1 city experiences the lower growth rate. As a consequence, since we assume Cobb-Douglas technologies, the type 3 city is characterized by a growth rate which is a convex combination of the two formers. This implies that the relative size in terms of output of the "agricultural" city decreases over time. This property allows to recover the fact that most nonagricultural production occurs in growing metropolitan areas. But, simultaneously, as we prove that total labor employed in each city is proportional to the total population, the relative population size distribution of cities is constant over time.

The rest of the paper is organized as follows. Section 2 presents the model and derives the optimality conditions. In section 3 we characterize the intertemporal equilibrium and we prove the existence of heterogeneous growth paths. Section 4 establishes the existence of a manifold of steady states and provides a local stability analysis showing saddle-point stability. Concluding comments are presented in section $5 .^{1}$

\section{The model}

There is a continuum $[0, N(t)]$ of infinitely-lived agents, each of them characterized by standard CRRA preferences such that

$$
\int_{0}^{+\infty} \frac{c(t)^{1-\theta}-1}{1-\theta} e^{-\rho t} d t
$$

where $c(t)$ is individual consumption at time $t, \theta>0$ is the inverse of the elasticity of intertemporal substitution in consumption and $\rho>0$ is the discount factor. Total population is assumed to grow at the constant exponential rate $n \in[0, \rho)$, so that $N(t)=e^{n t} N(0)$. Each agent supplies inelastically one unit of labor at each time $t$.

The economy consists of three cities, each performing different production functions. There are three factors of production, capital, $K(t)$, labor,

\footnotetext{
${ }^{1}$ All the proofs of the results are provided in the Working Paper version [10] available on the AMSE website at the address: https://www.amseaixmarseille.fr/en/research/working-papers.
} 
$L(t)$, and individual human capital, $h(t)$. We do not consider any externality. The numbers of workers $N_{1}(t), N_{2}(t)$ and $N_{3}(t)$ in these three cities together with their respective growth rates $\dot{N}_{1}(t) / N_{1}(t)=g_{N_{1}}(t)$, $\dot{N}_{2}(t) / N_{2}(t)=g_{N_{2}}(t)$ and $\dot{N}_{3}(t) / N_{3}(t)=g_{N_{3}}(t)$ are endogenously determined at the equilibrium. Types 1 and 2 cities produce intermediate goods, and capital and labor are freely mobile between them.

Type 1 produces an "agricultural intermediate good" using capital and labor only through the following Cobb-Douglas technology:

$$
Y_{1}(t)=L_{1}(t)^{\alpha_{1}} K_{1}(t)^{1-\alpha_{1}}
$$

Type 2 produces an "industrial intermediate good" using capital, labor and human capital. Agents working in this city devote a fraction $u(t) \in(0,1)$ of their unit of time to production. Total labor in this sector is therefore $L_{2}(t)=u(t) N_{2}(t)$. The rest of time $1-u(t)$ is devoted to the accumulation of individual human capital $h(t)$. Similarly to how Lucas [11] treats newborns, in our model each agent entering the type 2 city at any time $t_{0}$ acquires the available knowledge $h\left(t_{0}\right)$. We assume that human capital is accumulated as in Lucas [11], i.e.

$$
\dot{h}(t)=z[1-u(t)] h(t)
$$

with $z>0$. On the other hand, agents working in the type 1 city will spend all their unit of time to work so that total labor in this sector is $L_{1}(t)=N_{1}(t)$. The production of the "industrial intermediate good" is obtained through the following Cobb-Douglas technology:

$$
Y_{2}(t)=\left(L_{2}(t) h(t)\right)^{\alpha_{2}} K_{2}(t)^{1-\alpha_{2}}
$$

The product $L_{2}(t) h(t)=u(t) N_{2}(t) h(t)$ then represents total efficient labor. Contrary to the "agricultural" city, the "industrial" city employs more specialized workers, and is characterized by a higher productivity of labor.

Type 3 city produces the final good, which is consumed and used as capital in type 1 and 2 cities, using the two intermediate goods and labor. The technology is the following: ${ }^{2}$

\footnotetext{
${ }^{2}$ Some human capital could also be introduced as an input in the production of the final good, i.e. $Y(t)=Y_{1}(t)^{\beta_{1}} Y_{2}(t)^{\beta_{2}}\left(L_{3}(t) h(t)\right)^{1-\beta_{1}-\beta_{2}}$ with $L_{3}(t)=v(t) h(t), v(t) \in(0,1)$ and $\dot{h}(t)=z[1-u(t)-v(t)] h(t)$ but our main results would not be affected.
} 


$$
Y(t)=Y_{1}(t)^{\beta_{1}} Y_{2}(t)^{\beta_{2}} L_{3}(t)^{1-\beta_{1}-\beta_{2}}
$$

Capital is therefore freely mobile between types 1 and 2 cities, while labor is freely mobile between the three types of cities. ${ }^{3}$ Total capital is given by

$$
K(t)=K_{1}(t)+K_{2}(t)
$$

while total labor is

$$
L(t)=L_{1}(t)+L_{2}(t)+L_{3}(t)=N_{1}(t)+N_{2}(t) u(t)+N_{3}(t)
$$

The capital accumulation equation is standard

$$
\dot{K}(t)=Y(t)-\delta K(t)-L(t) c(t)
$$

where $\delta>0$ is the depreciation rate of capital. The optimization problem of the central planner is then: ${ }^{4}$

$$
\begin{array}{cl}
\max _{c(t), K_{1}(t), K_{2}(t), L_{1}(t), L_{2}(t), L_{3}(t), u(t), h(t)} & \int_{0}^{+\infty} \frac{c(t)^{1-\theta}-1}{1-\theta} e^{-(\rho-n) t} d t \\
\text { s.t. } & (2),(3),(4),(5),(6),(7),(8) \\
& K(0), h(0) \text { given }
\end{array}
$$

The Hamiltonian in current value is (we omit subscript for $t$ to simplify notations)

$$
\begin{aligned}
\mathcal{H} & =\frac{c^{1-\theta}-1}{1-\theta} \\
& +P\left[L_{1}^{\beta_{1} \alpha_{1}} K_{1}^{\beta_{1}\left(1-\alpha_{1}\right)}\left(L_{2} h\right)^{\beta_{2} \alpha_{2}}\left(K-K_{1}\right)^{\beta_{2}\left(1-\alpha_{2}\right)}\left(L-L_{1}-L_{2}\right)^{1-\beta_{1}-\beta_{2}}\right. \\
& -\delta K-N c]+Q z(1-u) h
\end{aligned}
$$

where $P$ is the utility price of the capital good and $Q$ the utility price of human capital. Applying the Pontryagin maximum principle allows to derive the following result:

\footnotetext{
${ }^{3}$ To simplify the analysis we assume that there is no transportation costs for goods and factors across cities. Introducing symmetric costs would not change our main results.

${ }^{4}$ As total population is growing at the exogenous growth rate $n$, we have indeed

$$
\int_{0}^{+\infty} N(t) \frac{c(t)^{1-\theta}-1}{1-\theta} e^{-\rho t} d t=N(0) \int_{0}^{+\infty} \frac{c(t)^{1-\theta}-1}{1-\theta} e^{-(\rho-n) t} d t
$$
}


Proposition 1. The optimal input demand functions and the optimal production level of the final good are respectively given by

$$
\begin{aligned}
K_{1} & =\frac{\beta_{1}\left(1-\alpha_{1}\right)}{\beta_{1}\left(1-\alpha_{1}\right)+\beta_{2}\left(1-\alpha_{2}\right)} K \equiv A_{1} K, K_{2}=\frac{\beta_{2}\left(1-\alpha_{2}\right)}{\beta_{1}\left(1-\alpha_{1}\right)+\beta_{2}\left(1-\alpha_{2}\right)} K \equiv A_{2} K, \\
L_{1} & =\frac{\beta_{1} \alpha_{1}}{1-\beta_{1}\left(1-\alpha_{1}\right)-\beta_{2}\left(1-\alpha_{2}\right)} L \equiv B_{1} L, L_{2}=\frac{\beta_{2} \alpha_{2}}{1-\beta_{1}\left(1-\alpha_{1}\right)-\beta_{2}\left(1-\alpha_{2}\right)} L \equiv B_{2} L, \\
L_{3} & =\frac{1-\beta_{1}-\beta_{2}}{1-\beta_{1}\left(1-\alpha_{1}\right)-\beta_{2}\left(1-\alpha_{2}\right)} L \equiv B_{3} L
\end{aligned}
$$

Moreover, there exists a function $v: \mathbb{R}^{5} \rightarrow(0,1)$ such that

$$
u(t)=v(K(t), h(t), P(t), Q(t), N(t))
$$

and we derive: ${ }^{5}$

$$
\begin{aligned}
N_{1}(t) & =\frac{B_{1} N(t) u(t)}{u(t)+B_{2}(1-u(t))}, N_{2}(t)=\frac{B_{2} N(t)}{u(t)+B_{2}(1-u(t))}, N_{3}(t)=\frac{u(t)\left(1-B_{1}-B_{2}\right) N(t)}{u(t)+B_{2}(1-u(t))} \\
L(t) & =\frac{u(t) N(t)}{u(t)+B_{2}(1-u(t))} \equiv \ell(K(t), A(t), P(t), Q(t), N(t)) N(t)
\end{aligned}
$$

together with the optimal production levels

$$
\begin{aligned}
Y_{1}(t)= & D_{1} K(t)^{1-\alpha_{1}}[\ell(K(t), A(t), P(t), Q(t), N(t)) N(t)]^{\alpha_{1}} \\
Y_{2}(t)= & D_{2} K(t)^{1-\alpha_{2}}(t) h^{\alpha_{2}}[\ell(K(t), A(t), P(t), Q(t), N(t)) N(t)]^{\alpha_{2}} \\
Y(t)= & D K(t)^{\beta_{1}\left(1-\alpha_{1}\right)+\beta_{2}\left(1-\alpha_{2}\right)} h(t)^{\beta_{2} \alpha_{2}}[\ell(K(t), A(t), P(t), Q(t), N(t)) N(t)]^{1-\beta_{1}\left(1-\alpha_{1}\right)-\beta_{2}\left(1-\alpha_{2}\right)} \\
& \text { with }
\end{aligned}
$$

$$
D_{1}=A_{1}^{1-\alpha_{1}} B_{1}^{\alpha_{1}}, D_{2}=A_{2}^{1-\alpha_{2}} B_{2}^{\alpha_{2}}, D=D_{1}^{\beta_{1}} D_{2}^{\beta_{2}}\left(1-B_{1}-B_{2}\right)^{1-\beta_{1}-\beta_{2}}
$$

Proposition 1 implies that the relative allocation of labor across cities is time-invariant as all labor demand functions $L_{i}, i=1,2,3$, are linear functions of total labor $L$. As we will discuss later on, this property is in line with the conclusions of Eeckout [8] (see also Soo [13] and Eaton and Eckstein [7]) showing that the relative populations of cities are stable over time despite the occurrence of successive industrialization and urbanization periods.

From the Pontryagin maximum principle, we also get the following differential equations for prices and stocks:

\footnotetext{
${ }^{5}$ In general, along an equilibrium path, it might be possible that $N_{1}(t)$ and/or $N_{3}(t)$ decrease. In this case, as noted before, agents that migrate from type 1 and 3 cities to type 2 city inherit the available individual stock of human capital.
} 


$$
\begin{aligned}
\dot{P} & =-P\left[\beta_{2}\left(1-\alpha_{2}\right) \frac{Y}{K_{2}}-\delta-\rho+n\right] \\
\dot{Q} & =-Q(z+n-\rho) \\
\dot{K} & =Y(t)-\delta K(t)-L(t) c(t) \\
\dot{h} & =z(1-u)
\end{aligned}
$$

It follows that any path, from any given initial conditions $(K(0), h(0))$, that satisfies Proposition 1, the conditions (12)-(15) and the transversality conditions

$$
\lim _{t \rightarrow+\infty} P(t) K(t) e^{-(\rho-n) t}=\lim _{t \rightarrow+\infty} Q(t) h(t) e^{-(\rho-n) t}=0
$$

is an optimal solution of problem (9).

\section{$3 \quad$ Heterogeneous growth paths}

Because human capital affects cities in an asymmetric way, we can derive the existence of city growth paths characterized by heterogeneous growth rates. Indeed, human capital enters only in the production of the "industrialized" good. Let us then define a heterogeneous growth path (HGP) as the state where all the variables grow at constant rates which are heterogeneous across cities. We thus rule out paths with ever increasing growth rates which will not satisfy the transversality conditions. Let us define the following growth rates:

$$
g_{c}=\frac{\dot{c}}{c}, g_{K}=\frac{\dot{K}}{K}, g_{K_{i}}=\frac{\dot{K}_{i}}{K_{i}}, g_{Y}=\frac{\dot{Y}}{Y}, g_{Y_{i}}=\frac{\dot{Y}}{Y_{i}}, g_{h}=\frac{\dot{h}}{h}
$$

We also introduce the following restrictions which guarantee positiveness of growth rates and interiority of the share $u$ of time devoted to work by agents in the type 2 city:

Assumption 1. $z>\rho-n$ and $\theta>1-(\rho-n) \frac{1-\beta_{1}\left(1-\alpha_{1}\right)-\beta_{2}\left(1-\alpha_{2}\right)}{z \beta_{2} \alpha_{2}}$.

From Proposition 1 and the differential equations (12)-(15) we derive:

Proposition 2. Let Assumption 1 hold. There exists a unique set of heterogeneous growth rates (HGR) such that 


$$
\begin{aligned}
& g_{Y}=g_{K}=g_{K_{1}}=g_{K_{2}}=g_{c}+n=\frac{(z+n-\rho) \beta_{2} \alpha_{2}}{1-\beta_{1}\left(1-\alpha_{1}\right)-\beta_{2}\left(1-\theta \alpha_{2}\right)}+n \\
& g_{h}=\frac{(z+n-\rho)\left[1-\beta_{1}\left(1-\alpha_{1}\right)-\beta_{2}\left(1-\alpha_{2}\right)\right]}{1-\beta_{1}\left(1-\alpha_{1}\right)-\beta_{2}\left(1-\theta \alpha_{2}\right)} \\
& g_{N_{1}}=g_{N_{2}}=g_{N_{3}}=n \\
& g_{Y_{1}}=\frac{(z+n-\rho)\left(1-\alpha_{1}\right) \beta_{2} \alpha_{2}}{1-\beta_{1}\left(1-\alpha_{1}\right)-\beta_{2}\left(1-\theta \alpha_{2}\right)}+n, g_{Y_{2}}=\frac{(z+n-\rho) \alpha_{2}\left[1-\beta_{1}\left(1-\alpha_{1}\right)\right]}{1-\beta_{1}\left(1-\alpha_{1}\right)-\beta_{2}\left(1-\theta \alpha_{2}\right)}+n \\
& g_{P}=-\theta g_{K}-n(1-\theta), g_{Q}=-(z+n-\rho)
\end{aligned}
$$

with $g_{Y_{2}}>g_{Y}>g_{Y_{1}}$. Moreover, along the HGP the amount of time devoted to production in the type 2 city is constant and given by

$$
u^{*}=\frac{z-g_{A}}{z}=\frac{1}{z}\left[\frac{z \beta_{2} \alpha_{2}(\theta-1)+(\rho-n)\left[1-\beta_{1}\left(1-\alpha_{1}\right)-\beta_{2}\left(1-\alpha_{2}\right)\right]}{1-\beta_{1}\left(1-\alpha_{1}\right)-\beta_{2}\left(1-\theta \alpha_{2}\right)}\right] \in(0,1)
$$

We can derive a number of important implications from Propositions 1 and 2. First, along the HGP, $g_{Y_{2}}>g_{Y}>g_{Y_{1}}$ and the growth rate $g_{Y}$ of output of the type 3 city is a convex combination of the growth rates of outputs in the "agricultural" and "industrial" cities and the growth rate of population, namely

$$
g_{Y}=\beta_{1} g_{Y_{1}}+\beta_{2} g_{Y_{2}}+\left(1-\beta_{1}-\beta_{2}\right) n
$$

This property shows that nonagricultural cities grow faster and allows to recover the fact that most nonagricultural production in developed countries occurs in growing metropolitan areas (see Black and Henderson [3]). Following the argument of Eaton and Eckstein [7], our assumption that each agent entering the type 2 city at any time $t_{0}$ acquires the available knowledge $h\left(t_{0}\right)$ is in line with the fact that migrants moving toward a dominant faster growing city would tend to be less educated than average upon arrival, but would acquire human capital more quickly once they arrive.

The accumulation of human capital in the type 2 city is used as labor augmenting technological progress in this city. Human capital accumulation then leads to an unbounded increase of TFP in the type 2 city and to capital deepening. The relative price of the "industrial" good decreases because of human capital accumulation while the relative price of the "agricultural" good produced in the type 1 city increases. This change of prices is associated with changes of demand of both intermediate goods by the type 3 
city. This process leads to endogenous growth of physical and human capital which drives the growth process of the three cities but with differences in the growth rates.

Second, the ratios $K_{1} / K_{2}, L_{1} / L_{2}, L_{1} / L_{3}$ and $L_{2} / L_{3}$ are constant, which is a consequence of the Cobb-Douglas technologies. Although the "industrial" city is asymptotically dominant in output, the amount of inputs used are not vanishing and labor does not shrink. As a result, the labor $L_{1}, L_{2}$ and $L_{3}$ in the three cities are growing at the same rate $n$ as total population $N$. Moreover, as shown in Proposition 1, the ratio $N_{1} / N_{3}$ is constant while the ratios $N_{1} / N_{2}$ and $N_{3} / N_{2}$ are proportional to the share $u$ which is constant along the HGP. As mentioned previously, this property is in line with the conclusions of Eeckout [8] or Eaton and Eckstein [7]. They show indeed that the relative populations of the main urban areas of cities, in particular in France and in Japan, are very stable over time despite the occurrence of successive industrialization and urbanization periods. Such a conclusion appears to be quite robust across developed countries and times periods as shown in Soo [13]. ${ }^{6}$

In order to study the stability properties of the HGP, we need to define the intertemporal equilibrium through a stationarized dynamical system characterized by a steady state. Using the normalization of variables as introduced by Caballe and Santos [5], we then define a stationarized HGP as follows: $k(t)=K(t) e^{-g_{K} t}, \eta(t)=h(t) e^{-g_{h} t}, p(t)=P(t) e^{-g_{P} t}$ and $q(t)=Q(t) e^{-g_{Q} t}$, for all $t \geq 0$, with $k(t), \eta(t), p(t)$ and $q(t)$ the stationarized values for $K(t), h(t), P(t)$ and $Q(t)$. As the price of human capital $Q$ is characterized by a linear differential equation with a constant growth rate $g_{Q}$, the solution of (13) is given by $Q(t)=Q(0) e^{-g_{Q} t}$ and its stationarized value is constant with $\dot{q}(t)=0$. We then get $q(t)=q(0)=q_{0}$ for all $t \geq 0$, and the initial value $Q(0)=q(0)=q_{0}$ will be chosen in order to select the optimal path. Recall that as population is growing at the exponential rate

\footnotetext{
${ }^{6}$ Other theoretical mechanisms to explain this stylized fact have been provided recently in the literature, see for instance Berliant and Watanabe [2], Rossi-Hansberg and Wright $[12]$.
} 
$n$, we have $N(t)=e^{n t} N(0)$ with $N(0)=N_{0}$ given.

Substituting these new variables into the dynamical system as given by equations (12)-(15) allows to obtain an equivalent stationarized system of differential equations which characterizes the equilibrium path.

Proposition 3. For any given $q_{0}>0$, the stationarized HGP is solution of the following dynamical system

$$
\begin{aligned}
\frac{\dot{p}}{p} & =-\left[F k^{\beta_{1}\left(1-\alpha_{1}\right)+\beta_{2}\left(1-\alpha_{2}\right)-1} \eta^{\beta_{2} \alpha_{2}} \ell\left(k, \eta, p, q_{0}, N_{0}\right)^{1-\beta_{1}\left(1-\alpha_{1}\right)-\beta_{2}\left(1-\alpha_{2}\right)}\right. \\
& \left.+g_{P}-\delta-\rho\right] \\
\frac{\dot{k}}{k} & =E k^{\beta_{1}\left(1-\alpha_{1}\right)+\beta_{2}\left(1-\alpha_{2}\right)-1} \eta^{\beta_{2} \alpha_{2}} \ell\left(k, \eta, p, q_{0}, N_{0}\right)^{1-\beta_{1}\left(1-\alpha_{1}\right)-\beta_{2}\left(1-\alpha_{2}\right)} \\
& -g_{K}-\delta-\frac{N_{0} p^{-\frac{1}{\theta}}}{k} \\
\frac{\dot{\eta}}{\eta} & =z\left[1-v\left(k, \eta, p, q_{0}, N_{0}\right)\right]-g_{h} \\
\text { with } E & =D N_{0}^{1-\beta_{1}\left(1-\alpha_{1}\right)-\beta_{2}\left(1-\alpha_{2}\right)} \text { and } F=\left[\beta_{1}\left(1-\alpha_{1}\right)+\beta_{2}\left(1-\alpha_{2}\right)\right] E .
\end{aligned}
$$

The transversality conditions (16) become

$$
\lim _{t \rightarrow+\infty} p(t) k(t) e^{-\left[\rho-n-\left(g_{K}-n\right)(1-\theta)\right] t}=\lim _{t \rightarrow+\infty} q_{0} h(t) e^{-\left(z-g_{h}\right) t}=0
$$

Since $\dot{h}=z(1-u) h$, we easily get $g_{h}=z(1-u)<z$ so that the second transversality condition always holds. Assumption 1 ensures that the first transversality condition also holds. Considering the stationarized dynamical system given in Proposition 3, we may now focus on the existence of a steady state and its local stability properties.

\section{Manifold of steady states and saddle-point sta- bility}

Once all the variables have been stationarized, we can look for the existence of a stationary equilibrium point of the system (19) which determines the stationary HGP. 
Proposition 4. For any given $q_{0}>0$, there exists a unique steady state $\left(p^{*}\left(q_{0}\right), k^{*}\left(q_{0}\right), \eta^{*}\left(q_{0}\right)\right)$ solution of the dynamical system (19). Moreover, $k^{*^{\prime}}\left(q_{0}\right)<0, \eta^{*^{\prime}}\left(q_{0}\right)<0$ and $p^{*^{\prime}}\left(q_{0}\right)>0$.

Proposition 4 proves that there exists a one-dimensional manifold of steady states for the capital stock $k$, human capital $\eta$ and the price of capital $p$ parameterized by the constant price of human capital $q_{0}$. The existence of a manifold of steady states in levels is standard in endogenous growth models (see for instance Lucas [11]) where the asymptotic equilibrium of the economy depends on the initial conditions. In our framework, for initial values of physical capital $k(0)$ and human capital $\eta(0)$, a unique value of $q_{0}$ will be selected in order for the economy to jump onto the optimal path and then converge to the particular steady state $\left(k^{*}\left(q_{0}\right), \eta^{*}\left(q_{0}\right), p^{*}\left(q_{0}\right)\right)$ situated on the manifold, thus satisfying all the equilibrium conditions.

To prove such a result, we need to study the local stability properties of the steady state. Linearizing the dynamical systems (19) around the steady state $\left(k^{*}\left(q_{0}\right), \eta^{*}\left(q_{0}\right), p^{*}\left(q_{0}\right)\right)$ for a given $q_{0}>0$, the local stability properties of $\left(k^{*}\left(q_{0}\right), \eta^{*}\left(q_{0}\right), p^{*}\left(q_{0}\right)\right)$ are appraised through the characteristic roots of the associated Jacobian matrix. Since we have two state variables, $k$ and $\eta$, and two forward variables, $p$ and $q$, with $q(t)=q_{0}$ being constant, the standard saddle-point stability occurs if there exists a one-dimensional stable manifold, i.e. if only one characteristic root is negative.

Theorem 1. Under Assumption 1, the steady state $\left(p^{*}\left(q_{0}\right), k^{*}\left(q_{0}\right), \eta^{*}\left(q_{0}\right)\right)$ is saddle-point stable and there exists a unique trajectory converging monotonously to the HGP.

In the dynamical system (19), the predetermined variables are the capital stock and the stock of human capital. For given initial conditions $K(0)=$ $k(0)=k_{0}$ and $h(0)=\eta(0)=\eta_{0}$, we generically cannot find a value of $q_{0}$ such that $\left(k_{0}, \eta_{0}\right)=\left(k^{*}\left(q_{0}\right), \eta^{*}\left(q_{0}\right)\right)$ and the economy is not in the set of HGP from the initial date. In other words, non-trivial transitional dynamics generically occurs starting from $\left(k_{0}, \eta_{0}\right) \neq\left(k^{*}, \eta^{*}\right)$. The initial values of the forward variables $p(0)=p_{0}$ and $q(0)=q_{0}$ are chosen such that the onedimensional stable optimal path converging toward a HGP is selected. As 
the stable manifold is one-dimensional, for any given $K(0)=k(0)=k_{0}$ and $h(0)=\eta(0)=\eta_{0}$ there exists a unique pair $\left(q_{0}, p_{0}\right)$ compatible with an equilibrium path. Of course, the choice of $q_{0}$ also determines the steady state on the manifold to which the equilibrium path will converge. The optimal value of $q_{0}$ will depend on the initial level of development as described by the pair $\left(k_{0}, \eta_{0}\right)$. A striking property is that, although the steady state values $\left(k^{*}\left(q_{0}\right), \eta^{*}\left(q_{0}\right), p^{*}\left(q_{0}\right)\right)$ depend on the selected $q_{0}$, the eigenvalues do not. It follows that the rate of convergence along any transitional path is the same, regardless of the initial conditions and thus of the asymptotic value of the steady state.

This property can be illustrated by the following Figure. It is easy to show that at the steady state $\eta^{*}$ and $k^{*}$ satisfy the following relationship as $q_{0}$ is varied:

$$
k^{*}=\mathcal{K} \ell^{*} \eta^{* \frac{\beta_{2} \alpha_{2}}{1-\beta_{1}\left(1-\alpha_{1}\right)-\beta_{2}\left(1-\alpha_{2}\right)}}
$$

with

$$
\mathcal{K}=\left[\frac{E\left[\beta_{1}\left(1-\alpha_{1}\right)+\beta_{2}\left(1-\alpha_{2}\right)\right]}{\theta\left(g_{K}-n\right)+\delta+\rho}\right]^{\frac{1}{1-\beta_{1}\left(1-\alpha_{1}\right)-\beta_{2}\left(1-\alpha_{2}\right)}}
$$

All pairs $\left(k^{*}, \eta^{*}\right)$ satisfying (20) correspond to a common asymptotic HGP but different optimal paths along the transition according to the initial condition $\left(k_{0}, \eta_{0}\right)$.

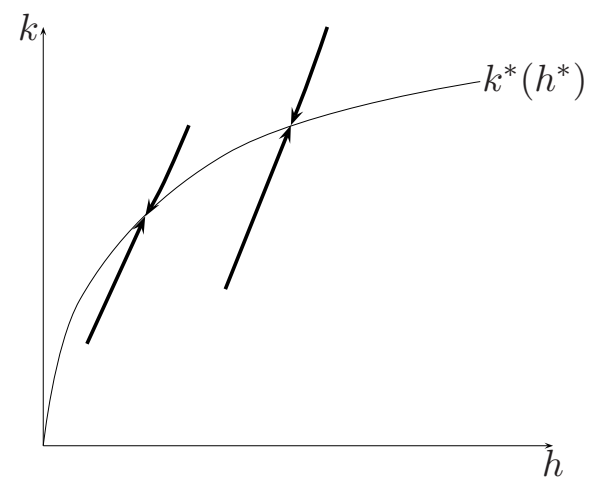

Figure 1: Manifold of steady states.

For a given pair $\left(k_{0}, \eta_{0}\right)$, the optimal path will converge toward an asymptotic position located on the curve $k^{*}\left(\eta^{*}\right)$ as given by (20) which depends on the initial position $\left(k_{0}, \eta_{0}\right)$ that defines the admissible initial value $q_{0}$. The 
arrows in Figure 1 illustrate some possible trajectories. Under these dynamics, then, a city beginning with low levels of human and physical capital will remain permanently below an initially better endowed city, and initial inequalities will persist over time (see Durlauf [6]).

The last important property is based on the fact that the convergence of the optimal path is monotonic. Indeed, it can be easily proved that the roots of the Jacobian matrix are real. It follows that, even though a priori more educated people from type 2 city could leave for the less educated type 1 and 3 cities, the monotonicity of the optimal paths together with the fact that the growth rate of the type 2 city is larger, workers will only move from type 1 and 3 cities to type 2 city in a monotonous way.

\section{Concluding comments}

We have considered an economy with three cities producing different outputs. Two cities produce intermediate goods, a type 1 producing an intermediate "agricultural" good with capital and labor only, and a type 2 producing an intermediate "industrial" good with capital, labor and human capital, and the last type 3 city produces the final good which is obtained from the two intermediate goods and labor. Following the strategy of Ghiglino et al. [9], this asymmetric introduction of human capital has allowed us to prove that the three cities experience at the equilibrium heterogeneous endogenous growth rates. As in Lucas, all the growth rates are proportional to the growth rate of human capital.

We have shown that the "industrial" type 2 city is characterized by the larger growth rate while the "agricultural" type 1 city experiences the lower growth rate, and thus the type 3 city is characterized by a growth rate which is a convex combination of the two formers. This implies that the relative size in terms of output of the "agricultural" city decreases over time. This property allows to recover the fact that most nonagricultural production occurs in growing metropolitan areas. But, simultaneously, as we prove that total labor employed in each city is proportional to the total population, the relative population size distribution of cities is constant over 
time.

\section{References}

[1] Acemoglu, D. and V. Guerrieri (2008): "Capital Deepening and NonBalanced Economic Growth," Journal of Political Economy, 116, 467498.

[2] Berliant, M. and H. Watanabe (2015): "Explaining the Size Distribution of Cities: Extreme Economies," Quantitative Economics 6, 153187.

[3] Black, D. and V. Henderson (1999): "Spatial Evolution of Population and Industry in the United States," The American Economic Review, Papers and Proceedings, 89, 321-327.

[4] Black, D. and V. Henderson (1999): "A Theory of Urban Growth," Journal of Political Economy, 107, 252-284.

[5] Caballe, J. and M. Santos (1993): "On Endogenous Growth with Physical and Human Capital," Journal of Political Economy, 101, 1042-1068.

[6] Durlauf, S. (1996): "A Theory of Persistent Income Inequality," Journal of Economic Growth, 1, 75-93.

[7] Eaton, J. and Z. Eckstein (1997): "Cities and Growth: Theory and Evidence from France and Japan," Regional Science and Urban Economics, $27,443-474$.

[8] Eeckhout, J. (2004): "Gibrat's Law for (all) Cities," American Economic Review 94, 1429-1451.

[9] Ghiglino, C., K. Nishimura and A. Venditti (2018): "Non-Balanced Endogenous Growth and Structural Change: When Romer Meets Kaldor and Kuznets," AMSE Working Paper 2018-31, Aix-Marseille School of Economics. 
[10] Ghiglino, C., K. Nishimura and A. Venditti (2019): "A Theory of Heterogeneous City Growth," AMSE Working Paper 2019, Aix-Marseille School of Economics, available at https://www.amseaixmarseille.fr/en/research/working-papers.

[11] Lucas, R. (1988): "On the Mechanics of Economic Development," Journal of Monetary Economics, 22, 3-42.

[12] Rossi-Hansberg, E. and M. Wright (2007): "Urban Structure and Growth," Review of Economic Studies, 74, 597-624.

[13] Soo, K. (2005): "Zipf's Law for Cities: A Cross-Country Investigation," Regional Science and Urban Economics, 35, 239-263. 